\title{
A Novel Binary Ant Colony Optimization: Application to the Unit Commitment Problem of Power Systems
}

\author{
Se-Hwan Jang*, Jae Hyung Roh ${ }^{\dagger}$, Wook Kim**, Tenzi Sherpa*, Jin-Ho Kim*** \\ and Jong-Bae Park*
}

\begin{abstract}
This paper proposes a novel binary ant colony optimization (NBACO) method. The proposed NBACO is based on the concept and principles of ant colony optimization (ACO), and developed to solve the binary and combinatorial optimization problems. The concept of conventional ACO is similar to Heuristic Dynamic Programming. Thereby ACO has the merit that it can consider all possible solution sets, but also has the demerit that it may need a big memory space and a long execution time to solve a large problem. To reduce this demerit, the NBACO adopts the state probability matrix and the pheromone intensity matrix. And the NBACO presents new updating rule for local and global search. The proposed NBACO is applied to test power systems of up to 100-unit along with 24-hour load demands.
\end{abstract}

Keywords: Binary ant colony optimization, Combinatorial optimization, Unit commitment, Swarm intelligence

\section{Introduction}

Ant Colony Optimization (ACO) is one of metaheuristic and evolutionary approaches to find the optimal solutions of the combinatorial or binary search problems. ACO was first developed by Dorigo et al. inspired by ant colonies [1,2]. In ACO, artificial ants search for good solutions in a cooperative way. Artificial ants move randomly along paths and deposit chemical substance trails, called pheromone, on the ground when they move, then collect and store information in pheromone trails. This pheromone trails motivates them to follow the path and can choose the shortest path in their movement. The ACO method has been researched in various aspects and successfully applied to the various optimization problems [3-10].

Unit commitment (UC) is a mixed integer combinatorial optimization problem, of which the purpose is to schedule the turning on and off of generating units so that the operating cost, start-up cost and shut-down cost be minimized for a given time horizon under various operating constraints [11]. The number of combinations of 0-1 variables grows exponentially as the number of units grows. Over the past decades, many salient methods have been developed for solving the UC problems. The exact solution to the problem can be obtained by complete enumeration, which cannot be applied to the real power systems due to its computational burden [11]. The solution methods for

$\dagger \quad$ Corresponding Author: Dept. of Electrical Engineering, Konkuk University, Korea. (jhroh@konkuk.ac.kr)

* Dept. of Electrical Engineering, Konkuk University, Korea (jhroh@konkuk.ac.kr)

** Korea Southern Power Co., Korea (dr.wookkim@gmail.com)

*** Dept. of Electrical Engineering, Kyungwon University, Korea. (kimjh@kyungwon.ac.kr)

Received: June 11, 2010; Accepted: September 1, 2010
UC problems can be divided into two categories: One is the numerical optimization techniques such as priority list methods [12-13], dynamic programming [14], Lagrangian relaxation methods [15], branch-and-bound methods [16], and mixed-integer programming [17]. The other is the stochastic methods based on heuristic search such as genetic algorithms [18], evolutionary programming [19], simulated annealing [20], particle swarm optimization [21], and quantum evolutionary algorithm [22].

Conventional ACO shows reasonable performance for small problems with moderate dimensions and searching space. However, Conventional ACO is recognized as not suitable for large scale problems such as UC problem, because the size of pheromone matrix grows exponentially along with the problem size [10]. This paper proposes a novel binary ant colony optimization (NBACO) method. The proposed NBACO is developed to solve the large size binary and combinatorial optimization problems. To overcome the computational burden of conventional ACO, the NBACO adopts the state probability matrix and the pheromone intensity matrix in place of the pheromone matrix used in conventional ACO method. In addition, the NBACO presents new updating rule for local and global search. The proposed NBACO is applied to UC problem of test power systems of up to 100-unit along with 24-hour load demands.

\section{Ant Colony Optimization Algorithm}

\subsection{Framework of Ant Colony System}

Entomologists have studied the ability of ants to find the 

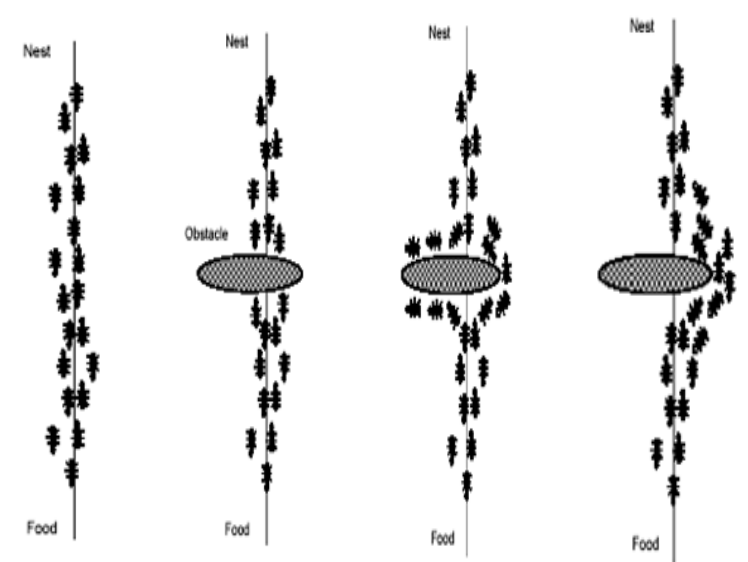

Fig. 1. An example of finding the shortest path.

shortest path between their nest and a food source. From these studies, Ant Colony Optimization (ACO) has been developed by Dorigo et al. [1,2] and successfully employed to solve various optimization problems. ACO is a metaheuristic and evolutionary approach where several generations of artificial ants in a cooperative way search for good solutions. Initially artificial ants move randomly along paths and deposit chemical substance trails, called pheromone, on the ground when they move. And ants collect and store information in pheromone trails during their moving. This pheromone trails motivate them to follow the path with high intensity of pheromone. With time, the pheromone trail is reinforced or evaporated by the move of ants. Finally, all ants can choose the shortest path in their movement.

\subsection{Ant Colony Optimization Algorithm}

As shown in Fig. 1, the agents (i.e. ants) are guided by the intensity of pheromone trails. The path rich in pheromone becomes the best tour with time. This concept inspired the ACO algorithm. Initially, each agent is positioned on a starting node. Agents move to feasible neighbor nodes following the state transition rule. This rule indicates the preference of ants in choosing their paths that connect the current node to the next node. During the moving process, ants modify the level of pheromone on the paths they choose by applying the local updating rule. If the pheromone level on the chosen paths is lowered, these paths become less attractive to other agents. This property gives agents a higher probability to explore different paths and find an improved solution. Once all agents have reached the final node and have identified the best path which has the optimal value of the objective function, they update the pheromone level on the best path by applying a global pheromone updating rule. This is intended to allocate a higher level of pheromone on the best path. The rules to find the best path are detailed as below:

\section{A. State Transition Rule}

This rule guides the agents' search toward neighbor nodes stochastically. The $k$-th agent at time $t$ positioned on node $r$ move to the next node $s$ along the shorter path with higher intensity of pheromone $\tau_{r u}(t)$. This is achieved by a state transition rule (1) that utilizes both the inverse of the length of the path $\eta_{r u}(t)$ and the amount of pheromone $\tau_{r u}(t)$

$$
s= \begin{cases}\arg \left\{\max _{u=\text { allowed }_{k}(t)}\left[\tau_{r u}(t)^{\alpha} \eta_{r u}^{\beta}\right]\right\} & \text { when } q \leq q_{0} \\ S & \text { otherwise }\end{cases}
$$

where,

$\tau_{r u}(t)$ : The pheromone trail at time $t$.

$\eta_{r u}(t)=1 / T C_{r s}(t)$ : the inverse of the transition cost $T C_{r s}(t)$ with $r s$ being the path from node $r$ at the current stage to node $s$ at the next stage

$\alpha, \beta$ : parameters representing the relative importance

$q \quad: \quad$ a random number uniformly distributed in $[0,1]$

$q_{0} \quad$ : a pre-specified parameter $\left(0 \leq q_{0} \leq 1\right)$ allowed $_{k(t)}$ : the set of feasible nodes currently not assigned by the ant $k$ at time $t$

$S \quad: \quad$ an index of node selected from allowed $_{k(t)}$ according to the probability distribution given by (2)

$$
P_{r s}^{k}(t)= \begin{cases}\frac{\tau_{r s}(t)^{\alpha} \eta_{r s}^{\beta}}{\sum_{u \in \text { allowed }_{k}(t)} \tau_{r u}(t)^{\alpha} \eta_{r u}^{\beta}} & \text { if } s \in \text { allowed }_{k}(t) \\ 0 & \text { otherwise }\end{cases}
$$

\section{B. Local Updating Rule}

An ant changes the pheromone level on the moved path (local updating) by applying the local updating rule (3). This rule has the effect of lowering the pheromone level on the search paths.

$$
\tau_{r s}=(1-\rho) \tau_{r s}+\rho \tau_{0}
$$

where,

$$
\begin{aligned}
& \rho: \text { evaporation coefficient }(0<\rho<1) \\
& \tau_{0} \quad \text { initial pheromone level, } \tau_{0}=1 / J \text { where } J \text { is a } \\
& \\
& \text { rough approximation of the optimum value of the } \\
& \text { cost function. }
\end{aligned}
$$

\section{Global Updating Rule}

The global pheromone updating is performed only after all ants have completed their moving. The global pheromone updating rule (4) is intended to provide a greater amount of pheromone to shorter path.

$$
\tau_{r s}=(1-\alpha) \tau_{r s}+\alpha \Delta \tau_{r e}
$$

where, 


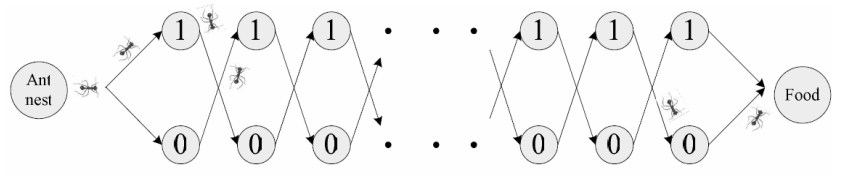

Fig. 2. Feasible paths of a binary ant colony optimization.

$$
\Delta \tau_{r e}=\left\{\begin{array}{cc}
\left(J^{*}\right)^{-1}, & \text { if }(r, s) \in \text { global }- \text { path } \\
0, & \text { otherwise }
\end{array}\right.
$$

$\alpha$ : pheromone decay coefficient $(0<\alpha<1)$

$J^{*}:$ the best value of the objective function

The capability of finding the optimal path can be enhanced through this rule in the search process. The pseudo code of Conventional ACO algorithm can be described as:

Initialize pheromone trails

Repeat until system convergence conditions satisfied Generate agents ( );

Move according to the transition Rules ( );

Update Pheromone ( );

End

\section{A Novel Binary Ant Colony Optimization}

\subsection{A novel Binary Ant Colony Optimization}

Fig. 2 presents feasible paths of a binary ant colony optimization for an integer optimization problem. Although the framework of NBACO is similar to the conventional $\mathrm{ACO}$, it adopts new rules and concepts to overcome the limit of conventional ACO. The new rules and concepts are described in the following subsections.

\section{A. State Transition Rule}

The proposed NBACO has a state probability matrix for all states. Using the state probability matrix, agents determine their own state. The state of each agent takes a value of 0 or 1 by the probability of $P_{i}^{k}$ of the state probability matrix as follows:

where,

$$
\mathbf{P}^{k}=\left[P_{1}^{k} \cdots P_{i}^{k} \cdots P_{H}^{k}\right]
$$

$P_{i}^{k} \quad$ : The probability that a state has value 1 at the stage i of $k$-trail

$H \quad$ : $\quad$ The number of the stages

$Q_{i}^{k}: \quad$ The probability that a state has value 0 at the stage i, $Q_{i}^{k}=1-P_{i}^{k}$

Initially, all states probability is $1 / 2$ and the state of each agent is determined by the state transition rule (6).

$$
S_{i}^{k}\left(R N, P_{i}^{k}\right)= \begin{cases}1, & \text { if } R N \leq P_{i}^{k} \\ 0, & \text { otherwise }\end{cases}
$$

where,

$$
R N \quad \text { : the random number }(0 \leq R N \leq 1)
$$

\section{B. Pheromone Updating Rule}

For pheromone updating, the relative Pheromone Intensity Matrix (7) is proposed as follows :

$$
\mathbf{C}^{k}=\left[C_{1}^{k} \cdots C_{i}^{k} \cdots C_{H}{ }^{k}\right], C_{i}^{k}=\sum_{j=1}^{N} c_{j}{ }^{k}
$$

where,

$$
\begin{aligned}
& c_{j}{ }^{k}=\left\{\begin{array}{ll}
+\rho, & s_{i}{ }^{k}=1 \\
-\rho, & s_{i}{ }^{k}=0
\end{array}, \mathbf{C}_{\text {initial }}{ }^{k}=0\right. \\
& N \text { : the number of agents } \\
& \rho \text { : an ant's pheromone quantity } \\
& H \text { : the number of the stages }
\end{aligned}
$$

It is assumed all agents move at the same time and they make relative pheromone intensity on the state of 0 or 1 . And pheromone intensity matrix is initialized to 0 at every trial (i.e., $\mathbf{C}_{\text {initial }}{ }^{k}=0$ ). As shown in equation (8), since relative pheromone intensity is divided by the number of the total agents (i.e., $N$ ), relative state selection probability (i.e., $\left.C_{i}^{t} / N\right)$ of $k$-trial is created. By a linear combination of both relative state selection probability and the best state, the state probability matrix is updated as follows:

$$
P_{i}^{k+1}=P_{i}^{k}+C_{i}^{k} / N+\rho \times \text { BState }_{i}^{k}
$$

where,

$$
P_{i}^{k+1}=\left\{\begin{array}{cc}
P_{i}^{k+1}, & 0<P_{i}^{k+1}<1 \\
1 \text { or } 0, & P_{i}^{k+1} \geq 1 \text { or } P_{i}^{k+1} \leq 0
\end{array}\right.
$$

BState $_{i}^{t}: k$-trail best state.

(the value of 0 state indicates -1 .)

$N$ : the number of agents

The procedure of the proposed NBACO algorithm can be summarized as the following pseudo-code:

$$
\begin{aligned}
& \text { Begin } \\
& \text { Create Initial State Probability Matrix } \\
& \text { Repeat until system convergence conditions satisfied } \\
& \text { Generate agents group ( ); } \\
& \text { Generate Relative Pheromone Intensity Matrix( ); } \\
& \text { Update Best State and State Probability Matrix ( ); } \\
& \text { End }
\end{aligned}
$$

\subsection{Application of NBACO to UC Problem}

The UC problem determines the on/off status of generating units. So the decision variables are the on/off states of generating units. The structure of an agent state of the proposed NBACO for UC problems is depicted in Fig. 3. If the $j$-th generator in $i$-th agent at hour $t$ of $k$-trial is ON, the 


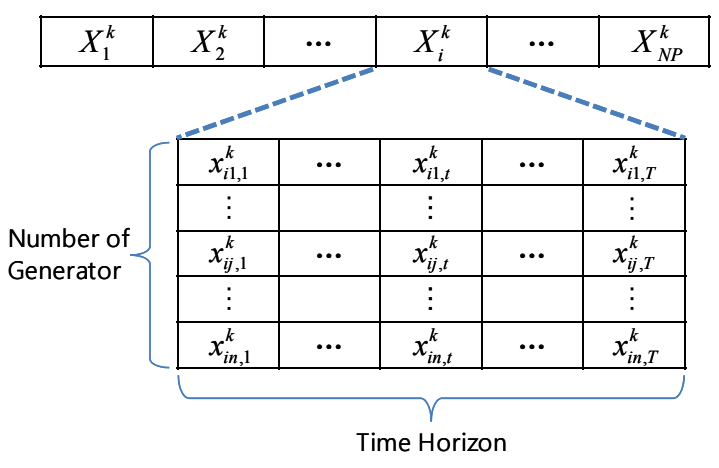

Fig. 3. The structure of an agent of NBACO for UC.

$x_{i j, t}^{k}$ is set to be 1 . Otherwise, $x_{i j, t}^{k}$ is set to be 0 .

After determining the feasible commitment scheduling, the optimal power outputs of the units are determined through the conventional economic dispatch (ED). Since the fuel cost function of a generating unit is represented as the quadratic function, ED problem can be easily solved by numerical techniques. In the subsequent sections, the detail procedures of NBACO for scheduling the on/off states of generating units are described.

\section{A. Creating Initial State Probability Matrix and Agents Group}

For UC problem, the state probability matrix at $k$-th trial is defined as follows:.

$$
P_{j t}^{k}=\left[\begin{array}{ccc}
P_{11} & \cdots & P_{1 H} \\
\vdots & \ddots & \vdots \\
P_{N 1} & \cdots & P_{N H}
\end{array}\right]
$$

The element of the matrix $P_{j t}^{k}$ represents the state probability that the $j$-th generator at hour $t$ of $k$-th trial is $1(\mathrm{ON})$. All elements of the state probability matrix are set to be $1 / 2$ at initial stage. It means that all states have the same probability. The state matrix of agents group on the $k$-th trial is determined by the state probability matrix (9) and the state transition rule (6). After generating a random number $R N_{i j, t}$, the state at the hour $t$ of the $j$-th generator at trial $k$ (i.e., $x_{i j, t}^{k}$ ) takes a value of 0 if $R N_{i j, t}$ is less than $1 / 2$, otherwise it is set to 1. All agents' states are generated in the same way.

\section{B. Creating Relative Pheromone Intensity Matrix}

The relative pheromone intensity matrix is generated by (10), which is composed of all agents' state. If the $x_{i j, t}^{k}$ is 1 , the pheromone's quantity (i.e., $\rho$ ) is added to relative pheromone intensity. Otherwise, it is subtracted. If an element of the matrix is positive, it means that the number of 1 states is larger than that of 0 states.

$$
\mathbf{C}^{k}=\left[C_{1}^{k} \cdots C_{j, t}^{k} \cdots C_{H}{ }^{k}\right], C_{j, t}^{k}=\sum_{i=1}^{N} c_{i j, t}^{k}
$$

where,

$$
c_{i j, t}^{k}=\left\{\begin{array}{ll}
+\rho, & x_{i j, t}^{k}=1 \\
-\rho, & x_{i j, t}^{k}=0
\end{array}, C_{\text {initial }}^{k}=0\right.
$$

\section{Updating the Best State}

If the state of $i$-th agent, $X_{i}^{k+1}$, yields a smaller cost function value than $B$ State $_{i}^{k}$, then $B$ State $e_{i}^{k+1}$ is set to $X_{i}^{k+1}$. Otherwise, $B$ State $_{i}^{k}$ is retained. The Rule Updating the best state can be formulated as (11).

$$
\text { BState }_{i}^{k+1}= \begin{cases}X_{i}^{k+1} & \text { if } f\left(X_{i}^{k+1}\right) \leq f\left(\text { BState }_{i}^{k}\right) \\ \text { BState }_{i}^{k} & \text { otherwise }\end{cases}
$$

\section{Updating the State Probability Matrix}

The state probability matrix is updated by (12), which is composed of the relative state selection probability matrix and the best state.

$$
P_{j t}^{k+1}=P_{j t}^{k}+C_{j, t}^{k} / N+\rho \times \text { BState }_{i}^{k}
$$

\section{E. Stopping Criteria}

The proposed NBACO algorithm is terminated if the iteration reaches a pre-specified maximum iteration or a prespecified critical probability.

\section{Numerical Test}

The proposed NBACO is applied to the several test systems, which have 10-, 20- 40-, 80-, and 100-generating units, respectively. Time horizon is chosen along with 24hour load demands. For each test case, 50 independent trials are conducted to compare the solution quality and convergence characteristics. Numerical tests have been executed on a Pentium IV $2.6 \mathrm{GHz}$ computer. In implementing the proposed algorithms, some parameters must be determined in advance. The number of agent and maximum iteration count are chosen same as those in the referred paper [22]. The value of pheromone quantity is obtained through a parameter tuning. When the pheromone quantity is more than 0.05 , the cost is observed to increase. In other words, the solution's quality becomes worse with higher pheromone quantity. In this paper the parameters were set through experiments as follows:

- The number of Agent $N=30$;

- Maximum iteration count iter $_{\max }=1,000$;

- Pheromone quantity $\rho=0.05$.

Firstly, the proposed NBACO is applied to the simple 10 -unit system with a 24-hour time horizon as a base case. 
Table 1. The generating units data for the 10-Unit system

\begin{tabular}{ccccccccccc}
\hline Unit & \multicolumn{1}{c}{ Unit1 } & Unit 2 & \multicolumn{1}{c}{ Unit 3} & Unit 4 & Unit 5 & Unit 6 & Unit 7 & Unit 8 & Unit 9 & Unit 10 \\
\hline$P_{\max }(\mathrm{MW})$ & 455 & 455 & 130 & 130 & 162 & 80 & 85 & 55 & 55 & 55 \\
$P_{\min }(\mathrm{MW})$ & 150 & 150 & 20 & 20 & 25 & 20 & 25 & 10 & 10 & 10 \\
$A$ & 1000 & 970 & 700 & 680 & 450 & 370 & 480 & 660 & 665 & 670 \\
$B$ & 16.19 & 17.26 & 16.6 & 16.5 & 19.7 & 22.26 & 27.74 & 25.92 & 27.27 & 27.79 \\
$C$ & 0.00048 & 0.00031 & 0.002 & 0.00211 & 0.00398 & 0.00712 & 0.00079 & 0.00413 & 0.00222 & 0.00173 \\
$M U T(\mathrm{Hr})$ & 8 & 8 & 5 & 5 & 6 & 3 & 3 & 1 & 1 & 1 \\
$M D T(\mathrm{Hr})$ & 8 & 8 & 5 & 5 & 6 & 3 & 3 & 1 & 1 & 1 \\
$S U_{H}(\$)$ & 4500 & 5000 & 550 & 560 & 900 & 170 & 260 & 30 & 30 & 30 \\
$S U_{C}(\$)$ & 9000 & 10000 & 1100 & 1120 & 1800 & 340 & 520 & 60 & 60 & 60 \\
$T_{\text {cold }}(\mathrm{Hr})$ & 5 & 5 & 4 & 4 & 4 & 2 & 2 & 0 & 0 & 0 \\
Initial status(Hr) & 8 & 8 & -5 & -5 & -6 & -3 & -3 & -1 & -1 & -1 \\
\hline
\end{tabular}

Table 2. The demand with 24-hour time horizon

\begin{tabular}{cc|cc|cc}
\hline Hour & Demand (MW) & Hour & Demand (MW) & Hour & Demand (MW) \\
\hline 1 & 700 & 9 & 1,300 & 17 & 1,000 \\
2 & 750 & 10 & 1,400 & 18 & 1,100 \\
3 & 850 & 11 & 1,450 & 19 & 1,200 \\
4 & 950 & 12 & 1,500 & 20 & 1,400 \\
5 & 1,000 & 13 & 1,400 & 21 & 1,300 \\
6 & 1,100 & 14 & 1,300 & 22 & 1,100 \\
7 & 1,150 & 15 & 1,200 & 23 & 900 \\
8 & 1,200 & 16 & 1,050 & 24 & 800 \\
\hline
\end{tabular}

Table 3. The simulation results of NBACO for test systems

\begin{tabular}{cccccc}
\hline Units & $\begin{array}{c}\text { Best Cost } \\
(\$)\end{array}$ & $\begin{array}{c}\text { Average Cost } \\
(\$)\end{array}$ & $\begin{array}{c}\text { Worst Cost } \\
(\$)\end{array}$ & $\begin{array}{c}\text { Standard } \\
\text { Deviation }\end{array}$ & $\begin{array}{c}\text { Execution } \\
\text { Time }(\mathrm{s})\end{array}$ \\
\hline 10 & 563,977 & 563,977 & 563,977 & 0 & 39 \\
\hline 20 & $1,124,531$ & $1,125,320$ & $1,126,251$ & 384 & 77 \\
\hline 40 & $2,246,022$ & $2,247,272$ & $2,248,710$ & 654 & 267 \\
\hline 80 & $4,487,254$ & $4,488,535$ & $4,489,890$ & 786 & 995 \\
\hline 100 & $5,609,583$ & $5,611,105$ & $5,612,892$ & 962 & 1535 \\
\hline
\end{tabular}

Table 4. The performance comparison of UC algorithms

\begin{tabular}{ccccc}
\hline Method & 10-unit & 40-unit & 80 -unit & 100-unit \\
\hline LR [15] & 565,825 & $2,258,503$ & $4,526,022$ & $5,657,277$ \\
GA [18] & 565,825 & $2,251,911$ & $4,504,933$ & $5,627,437$ \\
EP [19] & 564,551 & $2,249,093$ & $4,498,479$ & $5,623,885$ \\
SA [20] & 565,828 & $2,250,063$ & $4,498,076$ & $5,617,876$ \\
IPSO [21] & $\mathbf{5 6 3 , 9 5 4}$ & $2,248,163$ & $4,495,032$ & $5,619,284$ \\
NBACO & 563,977 & $\mathbf{2 , 2 4 6 , 0 2 2}$ & $\mathbf{4 , 4 8 7 , 2 5 4}$ & $\mathbf{5 , 6 0 9 , 5 8 3}$ \\
\hline
\end{tabular}

The characteristics of the generators used in the 10-unit system and the demand are given in Table 1 and Table 2, respectively. Subsequently, the 20-, 40-, 80-, and 100-unit data are obtained by duplicating the base case, and the load
Table 5. Unit scheduling and corresponding costs for the 10-unit system

\begin{tabular}{|c|c|c|c|c|c|c|c|c|c|c|c|c|c|}
\hline \multirow{2}{*}{$\mathrm{Hr}$} & \multicolumn{10}{|c|}{ Generation Ouput } & \multirow{2}{*}{$\begin{array}{l}\text { Total } \\
\text { Power }\end{array}$} & \multirow{2}{*}{$\begin{array}{l}\text { Fuel } \\
\text { Cost }\end{array}$} & \multirow{2}{*}{$\begin{array}{c}\text { Startup } \\
\text { Cost }\end{array}$} \\
\hline & $\mathrm{Gl}$ & $\mathrm{G} 2$ & $\mathrm{G} 3$ & G4 & G5 & G6 & G7 & G8 & G9 & $\mathrm{G} 10$ & & & \\
\hline 1 & 455 & 245 & 0 & 0 & 0 & 0 & 0 & 0 & 0 & 0 & 700 & 13,683 & 0 \\
\hline 2 & 455 & 295 & 0 & 0 & 0 & 0 & 0 & 0 & 0 & 0 & 750 & 14,554 & 0 \\
\hline 3 & 455 & 370 & 0 & 0 & 25 & 0 & 0 & 0 & 0 & 0 & 850 & 16,809 & 900 \\
\hline 4 & 455 & 455 & 0 & 0 & 40 & 0 & 0 & 0 & 0 & 0 & 950 & 18,598 & 0 \\
\hline 5 & 455 & 390 & 0 & 130 & 25 & 0 & 0 & 0 & 0 & 0 & 1,000 & 20,020 & 560 \\
\hline 6 & 455 & 360 & 130 & 130 & 25 & 0 & 0 & 0 & 0 & 0 & 1,100 & 22,387 & 1,100 \\
\hline 7 & 455 & 410 & 130 & 130 & 25 & 0 & 0 & 0 & 0 & 0 & 1,150 & 23,262 & 0 \\
\hline 8 & 455 & 455 & 130 & 130 & 30 & 0 & 0 & 0 & 0 & 0 & 1,200 & 24,150 & 0 \\
\hline 9 & 455 & 455 & 130 & 130 & 85 & 20 & 25 & 0 & 0 & 0 & 1,300 & 27,251 & 860 \\
\hline 10 & 455 & 455 & 130 & 130 & 162 & 33 & 25 & 10 & 0 & 0 & 1,400 & 30,058 & 60 \\
\hline 11 & 455 & 455 & 130 & 130 & 162 & 73 & 25 & 10 & 10 & 0 & 1,450 & 31,916 & 60 \\
\hline 12 & 455 & 455 & 130 & 130 & 162 & 80 & 25 & 43 & 10 & 10 & 1,500 & 33,890 & 60 \\
\hline 13 & 455 & 455 & 130 & 130 & 162 & 33 & 25 & 10 & 0 & 0 & 1,400 & 30,058 & 0 \\
\hline 14 & 455 & 455 & 130 & 130 & 85 & 20 & 25 & 0 & 0 & 0 & 1,300 & 27,251 & 0 \\
\hline 15 & 455 & 455 & 130 & 130 & 30 & 0 & 0 & 0 & 0 & 0 & 1,200 & 24,150 & 0 \\
\hline 16 & 455 & 310 & 130 & 130 & 25 & 0 & 0 & 0 & 0 & 0 & 1,050 & 21,514 & 0 \\
\hline 17 & 455 & 260 & 130 & 130 & 25 & 0 & 0 & 0 & 0 & 0 & 1,000 & 20,642 & 0 \\
\hline 18 & 455 & 360 & 130 & 130 & 25 & 0 & 0 & 0 & 0 & 0 & 1,100 & 22,387 & 0 \\
\hline 19 & 455 & 455 & 130 & 130 & 30 & 0 & 0 & 0 & 0 & 0 & 1,200 & 24,150 & 0 \\
\hline 20 & 455 & 455 & 130 & 130 & 162 & 33 & 25 & 10 & 0 & 0 & 1,400 & 30,058 & 490 \\
\hline 21 & 455 & 455 & 130 & 130 & 85 & 20 & 25 & 0 & 0 & 0 & 1,300 & 27,251 & 0 \\
\hline 22 & 455 & 455 & 0 & 0 & 145 & 20 & 25 & 0 & 0 & 0 & 1,100 & 22,736 & 0 \\
\hline 23 & 455 & 420 & 0 & 0 & 25 & 0 & 0 & 0 & 0 & 0 & 900 & 17,685 & 0 \\
\hline 24 & 455 & 345 & 0 & 0 & 0 & 0 & 0 & 0 & 0 & 0 & 800 & 15,427 & 0 \\
\hline Total & 10,920 & 9,680 & 2,080 & 2,210 & 1,540 & 332 & 225 & 83 & 20 & 10 & 27,100 & 559,887 & 4,090 \\
\hline
\end{tabular}

demands are adjusted in proportion to the system size. In all cases, the spinning reserve requirements are assumed to be $10 \%$ of the hourly demand.

In Table 3, the best, average, worst costs, standard deviation, and execution time for test systems obtained by the proposed NBACO algorithm are summarized.

The results of the proposed NBACO are compared with those of Lagrange relaxation (LR) [15], genetic algorithm (GA) [18], evolutionary programming (EP) [19], simulated annealing (SA) [20], and improved particle swarm optimization (IPSO) [21]. As shown in the Table 4, the results of the proposed algorithm are superior to those of other algorithms in most cases except the 10-unit case. Even in the 10 -unit case, the proposed method provides the second best solution, of which the difference to the best solution is negligible.

The commitment schedules during the planning horizon obtained by the proposed NBACO for 10-unit and 100-unit systems are described in Table 5 and Table 6, respectively. The convergence characteristics of the NBACO for 40-unit 
Table 6. Commitment scheduling for the 100-unit system

\begin{tabular}{|c|c|}
\hline $\mathrm{Hr}$ & Unit Schedule \\
\hline$\overline{1}$ & \\
\hline 2 & \\
\hline 3 & 111111111111111111110000000000000000000000000110000000000000000000000000000000000000000000000000000 \\
\hline 4 & 1111111111111111111110000000000000000000010111111110000000000000000000000000000000000000000000000000 \\
\hline 5 & 1111111111111111111111101000000010100010011111111110000000000000000000000000000000000000000000000000 \\
\hline 6 & 0101010100111111111100000 \\
\hline & 1111111111111111111111011101101101110111111111111100000000000000000000000000000000000000000 \\
\hline & 1111111111111111111111111111111111111111111111111111000000000000000 \\
\hline & 11111111111111111111111111111111111111110011111000111111100000000000000000000000000000000 \\
\hline & 111111111111 \\
\hline & 111111111111111111111111111111111111111111111111111111111111111111111101100110111001111110111001000 \\
\hline & 11111111111111111111111111111111111111111111111111 \\
\hline & 1111111111111111111111111111111111100110 \\
\hline & 1111111111111111111111111111111111111111111111111111111101011011010101101000000000000000000000000000000 \\
\hline & 1111111010111010111111111111111111000011000000100001000000000000000000000000000000 \\
\hline & 101110101111111111111 \\
\hline & 11111101011101011111111111111110000000000000000000000000000000000000000000000000 \\
\hline & 1111111010111010111111111111111110000000000000000000000000000000000000000000000000 \\
\hline & 1111111111110000000000111011010100000000000000000000000000000 \\
\hline & 11111111111111111111111111111111111111111111111111111111111111111100010100000000011111010000000 \\
\hline & 11111111111111111111011111111111111100111111101111111111111111111100000000000000000000000000000 \\
\hline & 111111111111111110011011100010110001000001111101111111111000100101000000000000000000000000000000 \\
\hline & 1111111111111111110000011100010100000000000000100000000000000000000000000000000000000000000000000 \\
\hline & 111111111111001111110000010100010100000000000000100000000000000000000000000000000000000000000000000000 \\
\hline
\end{tabular}

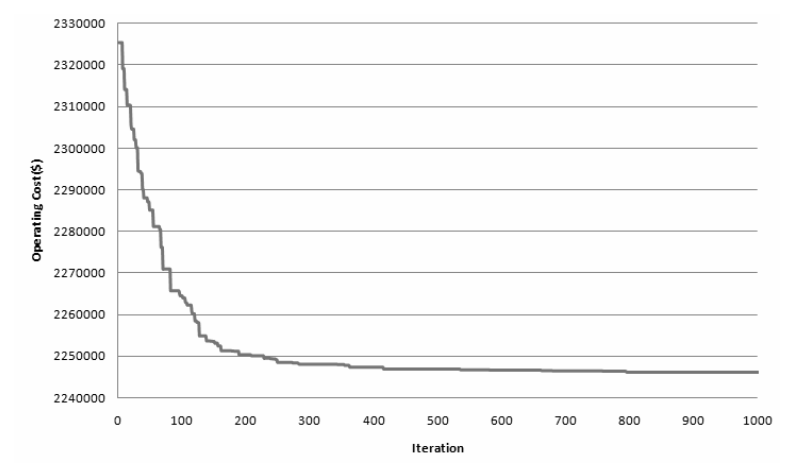

Fig. 4. Convergence characteristics of the NBACO for the 40-Unit system.

system are illustrated in Fig. 4.

\section{Conclusion}

This paper introduces a novel binary ant colony optimization (NBACO) approach and the proposed NBACO is applied to the Unit Commitment problems for power system. The proposed NBACO is based on the concept and principles of ant colony optimization (ACO), and developed to solve the binary and combinatorial optimization problems. The conventional ACO algorithm is known to have problems such as big memory requirement and long execution time. In order to reduce the restriction of the conventional ACO algorithm in solving a binary and combinatorial optimization problem, the proposed NBACO uses the state probability matrix and the pheromone intensity matrix. And the NBACO presents new update rule for local and global search. The proposed NBACO is applied to test power systems of up to 100 -unit along with 24-hour load demands and the results were compared with those of conventional optimization methods. The simulation results reveal that the proposed NBACO algorithm may provide better solution for UC problems than the conventional optimization methods in a reasonable time period.

\section{Appendix}

\section{A. Formulation of Unit Commitment Problem}

\section{1) Objective Function}

$$
\min \sum_{t=1}^{T} \sum_{j=1}^{N}\left[F_{j}\left(P_{j, t}\right) u_{j, t}+S U_{j, t}\left(1-u_{j, t-1}\right) u_{j, t}\right]
$$

where $T$ is the number of scheduling period, $N$ is the number of generating units, and $u_{j, t}$ is the on/off status of unit $j$ at hour $t$ (i.e., $u_{j, t}=1$ when unit $j$ is on-line, and $u_{j, t}=$ 0 when unit $j$ is off-line).

\section{Fuel Cost Function:}

$$
F_{j}\left(P_{j, t}\right)=a_{j}+b_{j} P_{j, t}+c_{j} P_{j, t}^{2}
$$

where $P_{j, t}$ is the power generation of unit $j$ at hour $t$ and $a_{j}, b_{j}, c_{j}$ are the cost coefficients of unit $j$.

\section{$\underline{\text { Start-up Cost: }}$}

$$
S U_{j, t}= \begin{cases}S U_{H, j} & \text { if } M D T_{j} \leq T O F F_{j, t} \leq M D T_{j}+T_{\text {cold }, j} \\ S U_{C, j} & \text { if } T O F F_{j, t}>M D T_{j}+T_{\text {cold }, j}\end{cases}
$$

where $S U_{H, j}$ and $S U_{C, j}$ are the hot and cold start cost. And $T_{\text {cold }, j}$ is the cold-start hour. TOFF $F_{j, t}$ is the duration for which unit $j$ has remained off-line at hour $t$ and $M D T_{j}$ is the minimum down-time of the $j$-th unit.

\section{2) System and Unit Constraints}

\section{Load Balance Constraints:}

$$
\sum_{j=1}^{N} P_{j, t} u_{j, t}=P D_{t}
$$

where $P D_{t}$ is the total system demand at hour $t$.

\section{Generation Limit Constraints:}

$$
u_{j, t} P_{j, \min } \leq P_{j, t} \leq u_{j, t} P_{j, \max }
$$

where $P_{j, \min }$ and $P_{j, \max }$ are the minimum and maximum 
generation limits of unit $j$, respectively.

\section{Spinning Reserve Constraints:}

$$
\sum_{j=1}^{N} P_{j, \max } u_{j, t} \geq P D_{t}+S R_{t}
$$

where $S R_{t}$ is the required spinning reserve at hour $t$.

\section{Generation Ramping Constraints:}

$$
R D_{j} \leq P_{j, t}-P_{j,(t-1)} \leq R U_{j}
$$

where $R D_{j}$ and $R U_{j}$ are the ramp-down and ramp-up limits of unit $j$, respectively.

\section{Minimum Up-time/Down-time Constraints:}

$$
u_{j, t}= \begin{cases}1 & \text { if } 1 \leq T O N_{j, t-1}<M U T_{j} \\ 0 & \text { if } 1 \leq T O F F_{j, t-1}<M D T_{j} \\ 0 \text { or } 1 & \text { otherwise }\end{cases}
$$

where $T O N_{j, t}$ is the duration for which unit $j$ remains online at hour $t$ and $M U T_{j}$ is the minimum up-time of unit $j$.

\section{References}

[1] M. Dorigo, "Optimization, learning, and natural algorithms," Ph.D. dissertation (in Italian), Dipartimento di Elettronica, Politecnico di Milano, Milano, Italy, 1992.

[2] M. Dorigo and L.M. Gambardella, "Ant colony system: a cooperative learning approach to the traveling salesman problem," IEEE Trans. on Evol. Compiut., Vol. 1, pp. 53-66, Apr. 1997.

[3] Christian Blum and Marco Dorigo, "The Hyper-Cube Framework for Ant Colony Optimization", IEEE Trans. Systems, Man and Cybernetics, Vol. 34, No. 2, pp. 1161-1172, April. 2004

[4] G. Wu and H. Huang, "Theoretical Framework of Binary Ant Colony Optimization Algorithm", IEEE Computer society, 2008

[5] S.-J. Huang, "Enhancement of hydroelectric generation scheduling using ant colony system based optimization approaches", IEEE Trans. Energy Conv., Vol. 16, No. 3, pp. 296-301, Mar. 2001.

[6] Y. H. Hou, Y. W. Wu, L. J. Lu and X. Y. Xiong, "Generlized Ant Colony Optimization for Economic Dispatch of Power Systems", IEEE, 2002.

[7] L. Shi, J. Hao, J. Zhou and G. Xu, "Ant colony optimisation algorithm with random perturbation behaviour to the problem of optimal unit commitment with probabilistic spinning reserve determination", Elec- tric Power Syst. Res., Vol. 69, pp. 295-303, 2004.

[8] S. P. Simon, N. P. Padhy and R. S. Anand, "An ant colony system for unit commitment problem," Elect. Power Energy Syst., 2006

[9] S. Chusanapiputt, D. Nualhong and S. Phoomvuthisarn, "Relativity Pheromone Updating Strategy in Ant Colony Optimization for Constrained Unit Commitment Problem", Power System Technology, IEEE, 2006

[10] Ahmed Yousuf Saber and Tomomobu Senjyu, "Memory-Bounded Ant Colony Optimization with Dynamic Programming and A* Local Search for Generator Planning", IEEE Trans. on Power Systems, Vol. 22, No. 4, pp. 1965-1973, Nov. 2007

[11] A. J. Wood and B. F. Wollenberg, Power Generation, Operation, and Control. New York: John Wiley \& Sons, Inc., 1984.

[12] R. M. Burns and C. A. Gibson, "Optimization of priority lists for a unit commitment program," in Proc. IEEE Power Engineering Society Summer Meeting, Paper A, 75 453-1, 1975.

[13] G. B. Sheble, "Solution of the unit commitment problem by the method of unit periods," IEEE Trans. on Power Systems, Vol. 5, No. 1, pp. 257-260, Feb. 1990.

[14] Z. Ouyang and S. M. Shahidehpour, "An intelligent dynamic programming for unit commitment application," IEEE Trans. on Power Systems, Vol. 6, No. 3, pp. 1203-1209, Aug. 1991.

[15] F. Zhuang and F. D. Galiana, "Toward a more rigorous and practical unit commitment by Lagrangian relaxation," IEEE Trans. on Power Systems, Vol. 3, No. 2, pp. 763-770, May 1988.

[16] A. I. Cohen and M. Yoshimura, "A branch-and-bound algorithm for unit commitment," IEEE Trans. on Power Apparatus and Systems, Vol. PAS-102, pp. 444-451, Feb. 1983.

[17] J. A. Muckstadt and R. C. Wilson, "An application of mixed-integer programming duality to scheduling thermal generating systems," IEEE Trans. on Power Apparatus and Systems, pp. 1968-1978, 1968

[18] S. A. Kazarlis, A. G. Bakirtzis, and V. Petridis, "A genetic algorithm solution to the unit commitment problem," IEEE Trans. on Power Systems, Vol. 11, No. 1, pp. 83-92, Feb. 1996.

[19] K. A. Juste, H. Kita, E. Tanaka, and J. Hasegawa, "An evolutionary programming solution to the unit commitment problem," IEEE Trans. on Power Systems, Vol. 14, pp. 1452-1459, Nov. 1999.

[20] D. N. Simopoulos, S. D. Kavatza, and C. D. Vournas, "Unit commitment by an enhanced simulated annealing algorithm," IEEE Trans. on Power Systems, Vol. 21, No. 1, pp. 68-76, Feb. 2006.

[21] B. Zhao, C. X. Guo, B. R. Bai and Y. J. Cao, "An improved particle swarm optimization algorithm for unit commitment," Electrical Power \& Energy Systems, Vol. 28, Issue 7, pp. 482-490, Sep. 2006.

[22] Y.-W. Jeong, J.-B. Park, J.-R. Shin, and K. Y. Lee, “A thermal unit commitment approach using an im- 
proved quantum evolutionary algorithm", Electric Power Components and Systems, Vol. 37, No. 7, pp. 770-786, July 2009.

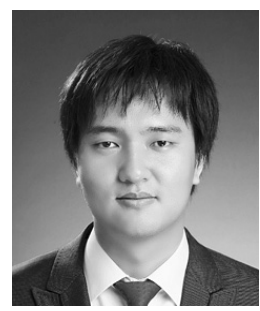

Se-Hwan Jang received his B.S. and M.S. degrees from Pusan National University of Korea in 2006 and 2008. And he is working on Ph.D. degree at Konkuk University. His research interest includes power system operation, planning, economics, market analysis and smart grid.

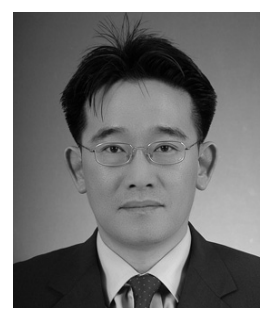

Jae Hyung Roh received the B.S. degree in Nuclear Engineering from Seoul National University, Korea, in 1993 and the M.S. degree in Electrical Engineering from Hongik University, Korea, in 2002. He received Ph.D. degree in Electrical Engineering from Illinois Institute of Technology, Chicago, USA. For 1992-2001, he was with Korea Electric Power Corporation, and for 2001-2010, he was with Korea Power Exchange. Since 2010, he has been with Electrical Engineering Department at Konkuk University, Seoul, as an Assistant Professor. His research interests include power systems restructuring, smart grid and resource planning.

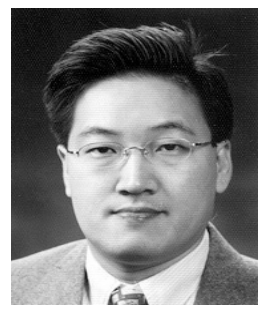

Wook Kim was born in Pusan, Korea on February 11, 1968. He received B.S., M.S. and Ph.D. degrees in Electrical Engineering from Seoul National University in 1990, 1992 and 1997, respectively. $\mathrm{He}$ is a manager of overseas business team at Korea Southern Power Co. His research interests include power economics, electricity and carbon trading, smart grid, renewable energy and generation expansion planning

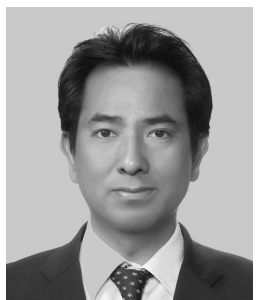

Tenzi Sherpa was born in Solukhumbu, Nepal on July 15, 1967. He received B.S. in Electronics Engineering and M.S. degree in Electrical Engineering from Seoul National University in 1993 and 1995 respectively. He worked at Hyundai Engineering Co., Ltd. from 1995 to 2009 as electrical engineer. His research interests include power economics, power development, Hydro-Power and renewable energy.

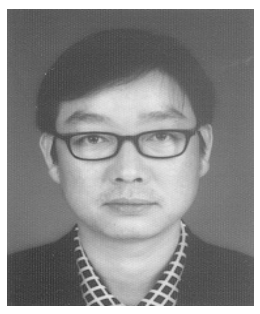

Jin-Ho, Kim received his $\mathrm{Ph}$. D degree from Seoul National University in 2001 and he is at Kyungwon University as an associate professor. His research interest includes optimal operation and planning of power system resources, deregulation and restructuring of power industry, electricity market analysis, and distribution and renewable energy sources.

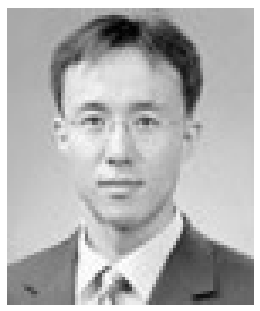

Jong-Bae Park (M'98) received B.S., M.S., and Ph.D. degrees from Seoul National University in 1987,1989 , and 1998, respectively. For 1989-1998, he was with Korea Electric Power Corporation, and for 1998-2001 he was an Assistant Professor at Anyang University, Korea. For 2006-2008, he was a guest researcher of EPRI, USA. From 2001, he has been with Electrical Engineering Department at Konkuk University as Professor. His major research topics include power system operation, planning, economics, and markets. 